\title{
The Molecular Structure of Trichodermin
}

\author{
SIXTEN ABRAHAMSSON and BO NILSSON \\ Crystallography Group, Department of Medical Biochemistry, University of Göteborg, \\ Göteborg, Sweden
}

\begin{abstract}
The structure of the antibiotic trichodermin has been determined by X-ray single crystal analysis of trichodermol $p$-bromobenzoate. A new type of ring system was discovered which has later been found to be present in several other natural products. The previously assumed structures of these compounds, which include verrucarol and trichotecin have therefore had to be revised.
\end{abstract}

$\mathrm{T}$

richodermin $\left(\mathrm{C}_{17} \mathrm{H}_{24} \mathrm{O}_{4}\right)$ is a new type of sesquiterpene antibiotic isolated from a soil sample from New Guinea. Godtfredsen and Vangedal have performed extensive chemical studies ${ }^{1,2}$ on the antibiotic. They showed that the compound was related to trichotecin, ${ }^{3,4}$ and trichodermin was therefore thought to have structure (I) with a four-membered oxygen containing ring suggested to be present in trichotecin.

Some reactions were, however, in conflict with such a structure. At the suggestion of Dr. Godtfredsen we have performed a single crystal analysis in order to establish definitely the molecular structure of the compound. We have earlier published a preliminary paper on the X-ray analysis. ${ }^{5}$

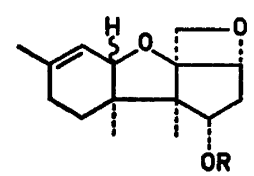

(I)

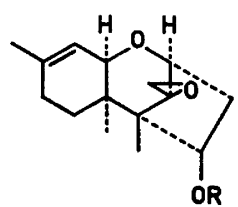

(II)

Trichodermin: $\mathbf{R}=$ acetyl

Trichodermol: $\mathbf{R}=\mathbf{H}$

\section{EXPERIMENTAL}

Good single crystals of the trichodermol $p$-bromobenzoate $\left(\mathrm{C}_{22} \mathrm{H}_{25} \mathrm{O}_{4} \mathrm{Br}\right)$ were kindly provided by Dr Godtfredsen. They are orthorhombic with

$$
a=10.69 \AA, b=9.24 \AA \text {, and } c=20.70 \AA
$$

Acta Chem. Scand. 20 (1966) No. 4 
(CuKa-radiation). The space group is $P 2_{1} 2_{1} 2_{1}$ and there are four molecules per unit cell. Weissenberg photographs were taken for layers $0-4$ about the $a$-axis and for layers 0-2 about [110]. The intensities were estimated visually and corrected for the Lorentz and polarization factors. No absorption correction was made.

\section{STRUCTURE DETERMINATION}

The structure was solved by the heavy-atom method. All atoms except hydrogens had been found after 5 cycles of Fourier refinement. The $R$-value was then 0.22. All Fourier series (including the Patterson synthesis) were scanned for peaks in the computer making possible a short cycle time. The oxygen atoms could be easily differentiated from the carbon atoms from the Fourier maps.

The structure was refined by full matrix least-squares techniques. The hydrogen atoms were included in the structure factor calculations with their expected coordinates and with isotropic temperature factors corresponding to those of the hydrogen carrying heavier atoms. The refinement was continued until the shifts were smaller than one fifth of the standard deviations. The final $R$-value for the 888 independent reflexions is 0.102 .

All calculations were performed on the Datasaab D21 computer with the integrated crystallographic programme system developed at this institute. ${ }^{6}$ The formfactors given in the International Tables for X-ray Crystallography,

Table 1. Fractional coordinates (with standard deviations) $\times 10^{4}$ for the heavier atoms of the molecule.

\begin{tabular}{|c|c|c|c|c|}
\hline & $\begin{array}{ll}x & \sigma(x)\end{array}$ & $y \quad \sigma(y)$ & $z$ & $\sigma(z)$ \\
\hline $\mathrm{Brl}$ & 1008 & $4871 \quad(2)$ & 1585 & (1) \\
\hline 01 & 1991 (15) & 4200 (11) & 4871 & (5) \\
\hline $\mathrm{O} 2$ & 2417 (14) & $1878(10)$ & 4546 & (4) \\
\hline O3 & 3267 (21) & 1792 (14) & 9802 & (5) \\
\hline 04 & 1835 (14) & 0560 (12) & 1373 & (4) \\
\hline $\mathrm{Cl}$ & $1446(23)$ & 4319 (18) & 2455 & (6) \\
\hline C2 & $1599(22)$ & 2956 (18) & 2611 & (6) \\
\hline C3 & $1903(28)$ & $2584(21)$ & 3265 & (7) \\
\hline $\mathrm{C4}$ & $1838(20)$ & 3612 (17) & 3702 & (6) \\
\hline C5 & $1603(23)$ & 5086 (16) & 3557 & (7) \\
\hline C6 & 3657 (25) & $4547(21)$ & 7912 & (7) \\
\hline C7 & $2001(22)$ & 3307 (16) & $\mathbf{4 4 2 8}$ & (6) \\
\hline C8 & $2574(19)$ & 1489 (15) & 5208 & (6) \\
\hline C9 & 1372 (22) & 0537 (16) & 5475 & (6) \\
\hline C10 & 3073 (24) & 1017 (19) & 0414 & (6) \\
\hline C11 & $1737(26)$ & 0850 (18) & 0684 & (6) \\
\hline $\mathrm{C} 12$ & 3717 (22) & 0432 (18) & 5267 & (8) \\
\hline Cl3 & 0252 (23) & $0805(24)$ & 5101 & (8) \\
\hline C14 & 3935 (35) & 2394 (17) & 0398 & (8) \\
\hline C15 & 1353 (17) & 0996 (14) & 6199 & (6) \\
\hline C16 & $2556(22)$ & 0791 (15) & 6540 & (7) \\
\hline C17 & 0838 (26) & 2489 (20) & 6287 & (7) \\
\hline $\mathrm{Cl8}$ & 0382 (24) & $-0140(18)$ & 6551 & (7) \\
\hline Cl9 & 0339 (26) & 0424 (20) & 7328 & (10) \\
\hline $\mathrm{C} 20$ & 1539 (22) & 0627 (20) & 7620 & (6) \\
\hline C21 & 2491 (20) & 0821 (15) & 7259 & (7) \\
\hline $\mathrm{C} 22$ & $1417(28)$ & $1001(25)$ & 8344 & (7) \\
\hline
\end{tabular}

Acta Chem. Scand. 20 (1966) No. 4 
Vol. III (pp. 202-207) were used. The weight assigned to each observation in the least-squares refinement was ${ }^{7}$

$$
w=\frac{1}{1+\left[\left(\left|F_{o}\right|-4\left|F_{\min }\right|\right) / 3\left|F_{\min }\right|\right]^{2}}
$$

\section{RESULTS AND DISCUSSION}

The positions for the heavier atoms used in the final calculations of the structure factors are given with standard deviations in Table 1. Allowance was made for anisotropic vibrations by a factor

$$
\begin{gathered}
\exp \left[-2 \pi^{2}\left(h^{2} a^{* 2} \mathrm{U}_{11}+k^{2} b^{* 2} \mathrm{U}_{22}+l^{2} c^{* 2} \mathrm{U}_{33}+2 k l b^{*} c^{*} \mathrm{U}_{23}\right.\right. \\
\left.\left.+2 l h c^{*} a * \mathrm{U}_{31}+2 h k a * b * \mathrm{U}_{12}\right)\right]
\end{gathered}
$$

(Table 2). Table 3 gives the parameters of the hydrogen atoms. Observed and calculated structure factors are listed in Table 4.

A composite drawing of the three-dimensional electron density series based on the final phases is given in Fig. 1. Fig. 2 illustrates the stereoche nistry

\begin{tabular}{|c|c|c|c|c|c|c|}
\hline & $U_{11}$ & $U_{22}$ & $\mathrm{U}_{33}$ & $\mathrm{U}_{23}$ & $\mathbf{U}_{31}$ & $\mathrm{U}_{12}\left(\AA^{2}\right)$ \\
\hline Brl & 3017 & 1016 & 0577 & 0195 & -0167 & 0255 \\
\hline 01 & 1893 & 0528 & 0642 & 0055 & -0163 & 0100 \\
\hline $\mathrm{O} 2$ & 2199 & 0522 & 0347 & 0117 & 0020 & 0181 \\
\hline 03 & 3537 & 0786 & 0546 & 0108 & -0201 & 0174 \\
\hline O4 & 1921 & 0695 & 0543 & -0123 & 0026 & 0183 \\
\hline Cl & 2356 & 0654 & 0364 & 0243 & 0048 & 0203 \\
\hline C2 & 1846 & 0751 & 0408 & -0041 & -0056 & 0162 \\
\hline C3 & 2375 & 0947 & 0534 & 0122 & -0037 & -0127 \\
\hline C4 & 1645 & 0724 & 0390 & -0054 & 0114 & 0197 \\
\hline C5 & 1878 & 0557 & 0687 & 0122 & 0177 & 0083 \\
\hline C6 & 2411 & 0843 & 0550 & -0187 & 0456 & 0255 \\
\hline C7 & 1934 & 0571 & 0471 & -0026 & 0015 & -0037 \\
\hline C8 & 1709 & 0438 & 0494 & 0173 & -0137 & 0206 \\
\hline C9 & 2219 & 0435 & 0495 & 0035 & 0016 & 0135 \\
\hline Clo & 2108 & 0936 & 0308 & 0096 & 0150 & -0214 \\
\hline Cl1 & 2794 & 0711 & 0359 & -0134 & -0180 & 0488 \\
\hline C12 & 1771 & 0603 & 0766 & 0218 & 0266 & -0081 \\
\hline C13 & 1723 & 1098 & 0618 & -0029 & 0241 & -0090 \\
\hline C14 & 3846 & 0421 & 0888 & -0169 & 0105 & -0132 \\
\hline C15 & 1158 & 0545 & 0452 & 0109 & 0133 & 0249 \\
\hline C16 & 2232 & 0443 & 0672 & -0159 & -0176 & 0211 \\
\hline Cl7 & 2224 & 0979 & 0457 & -0078 & -0202 & 0260 \\
\hline C18 & 2070 & 0950 & 0585 & 0105 & 0214 & -0523 \\
\hline C19 & 2000 & 0700 & 1111 & 0298 & 0613 & 0167 \\
\hline C20 & 1807 & 0897 & 0442 & -0078 & -0281 & 0157 \\
\hline C21 & 2073 & 0487 & 0456 & -0223 & -0126 & 0191 \\
\hline C22 & 2897 & 1284 & 0502 & 0229 & 0398 & 0857 \\
\hline
\end{tabular}
of trichodermol $p$-bromobenzoate. The numbering of atoms is given in Fig. 3 .

Table 2. $\mathrm{U}_{i j}: \mathrm{s} \times 10^{4}$ for the heavier atoms.

Acta Chem. Scand. 20 (1966) No. 4 


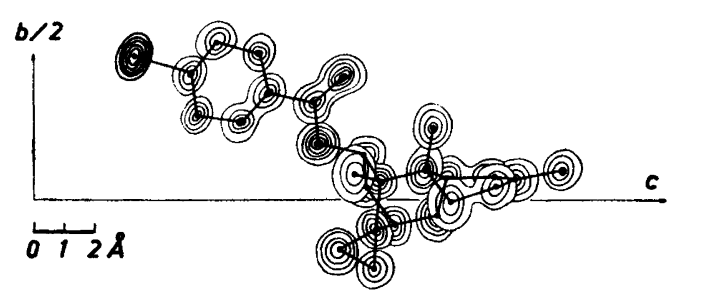

Fig. 1. Composite drawing of the three-dimensional electron density series of trichodermol $p$-bromobenzoate. Contours are given at intervals of 1 e $\AA^{-3}$ starting at 1 e $\AA^{-3}$ except for the bromine atom which is contoured at intervals of 5 e $\AA^{-3}$.

The X-ray analysis determines the molecular structure of trichodermol as (II). The ring system with the epoxy group is quite different from that of (I) based on the trichotecin structure. The chemical implications of this have been thoroughly discussed by Godtfredsen and Vangedal. ${ }^{2}$ The trichodermin ring system seems to be fairly widespread in nature. Godtfredsen and Vangedal suggested a revision of the earlier proposed molecular structures of trichotecin ${ }^{3,4}$ and verrucarol. ${ }^{8} \mathrm{~A}$ recent $\mathrm{X}$-ray analysis by $\mathrm{McPhail}$ and $\operatorname{Sim}^{9}$ of the $p$-iodobenzenesulphonate of verrucarin A also confirms the presence of the trichodermin ring system.

Table 3. Fractional coordinates $\times 10^{4}$ and isotropic temperature factors for the hydrogens of the molecule.

\begin{tabular}{|c|c|c|c|c|}
\hline & $x$ & $y$ & $z$ & $B\left(\AA^{2}\right)$ \\
\hline $\mathrm{H} 61$ & 1079 & 6548 & 2771 & 8.1 \\
\hline H51 & 1622 & 5909 & 3934 & 7.0 \\
\hline H31 & 2179 & 1487 & 3395 & 8.4 \\
\hline H21 & 1497 & 2112 & 2247 & 6.5 \\
\hline H161 & 3433 & 0628 & 6282 & 6.8 \\
\hline H81 & 2361 & 2340 & 5555 & 5.6 \\
\hline H211 & 3369 & 1036 & 7509 & 6.0 \\
\hline H121 & 4001 & 0053 & 4790 & 7.3 \\
\hline H122 & 4502 & 0975 & 5499 & 7.3 \\
\hline H111 & 4129 & -0934 & 5408 & 7.0 \\
\hline H191 & -0170 & -0381 & 7609 & 9.1 \\
\hline H192 & -0160 & 1452 & 7344 & 9.1 \\
\hline H181 & 0730 & -1247 & 6519 & 8.2 \\
\hline H182 & -0544 & -0080 & 6331 & 8.2 \\
\hline H141 & 0054 & -2290 & 5448 & 8.9 \\
\hline H142 & 1371 & $-\mathbf{3 4 0 4}$ & 5618 & 8.9 \\
\hline H221 & 1560 & 2159 & 8412 & 9.7 \\
\hline H222 & 2119 & 0404 & 8618 & 9.7 \\
\hline H223 & 0487 & 0704 & 8514 & 9.7 \\
\hline H171 & 0762 & 2728 & 6802 & 7.8 \\
\hline H172 & -0086 & 2555 & 6066 & 7.8 \\
\hline H173 & 1460 & 3271 & 6059 & 7.8 \\
\hline H131 & 0417 & 0511 & 4599 & 8.3 \\
\hline H132 & 0009 & 1949 & 5130 & 8.3 \\
\hline H133 & -0515 & 0158 & 5294 & 8.3 \\
\hline
\end{tabular}

Acta Chem. Scand. 20 (1966) No. 4 
Fig. 2. Stereochemistry of trichodermol $p$-bromobenzoate.

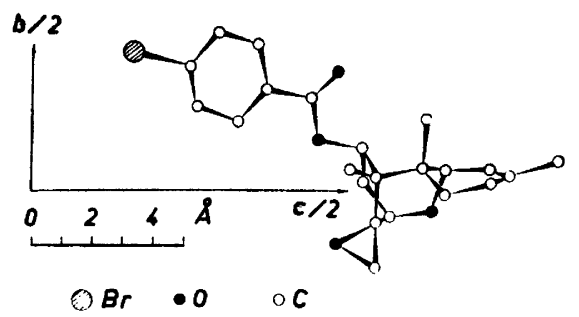

The distances and angles of trichodermol $p$-bromobenzoate are given in Fig. 3 and in Table 5 with the estimated standard deviations. The latter were calculated from the diagonal elements of the inverse least-squares matrix. ${ }^{10,11}$ The bond distances and angles show rather large deviations from the generally accepted values, but, considering the standard deviations, there are no anomalies in the structure. The average bond distance between single bonded carbons is $1.55 \AA, 1.39 \AA$ for the benzene ring carbons, and $1.46 \AA$ for the $\mathrm{C}-\mathrm{O}$ distance. The accuracy appears to be much the same as in other bromo derivatives of natural products investigated earlier at this institute, e.g. prostaglandin $\mathbf{F}_{2-1} p$-bromobenzoate. ${ }^{12}$
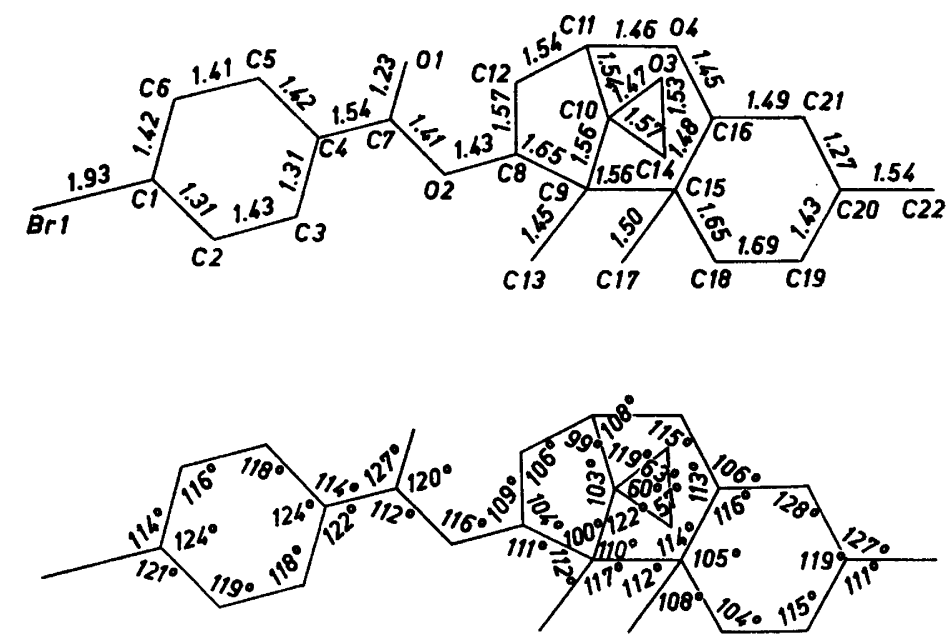

Fig. 3. Bond distances and bond angles for trichodermol $p$-bromobenzoate. 
Table 4. Observed and calculated structure factors $(\times 100)$ with phase angles as fractions of one revolution.

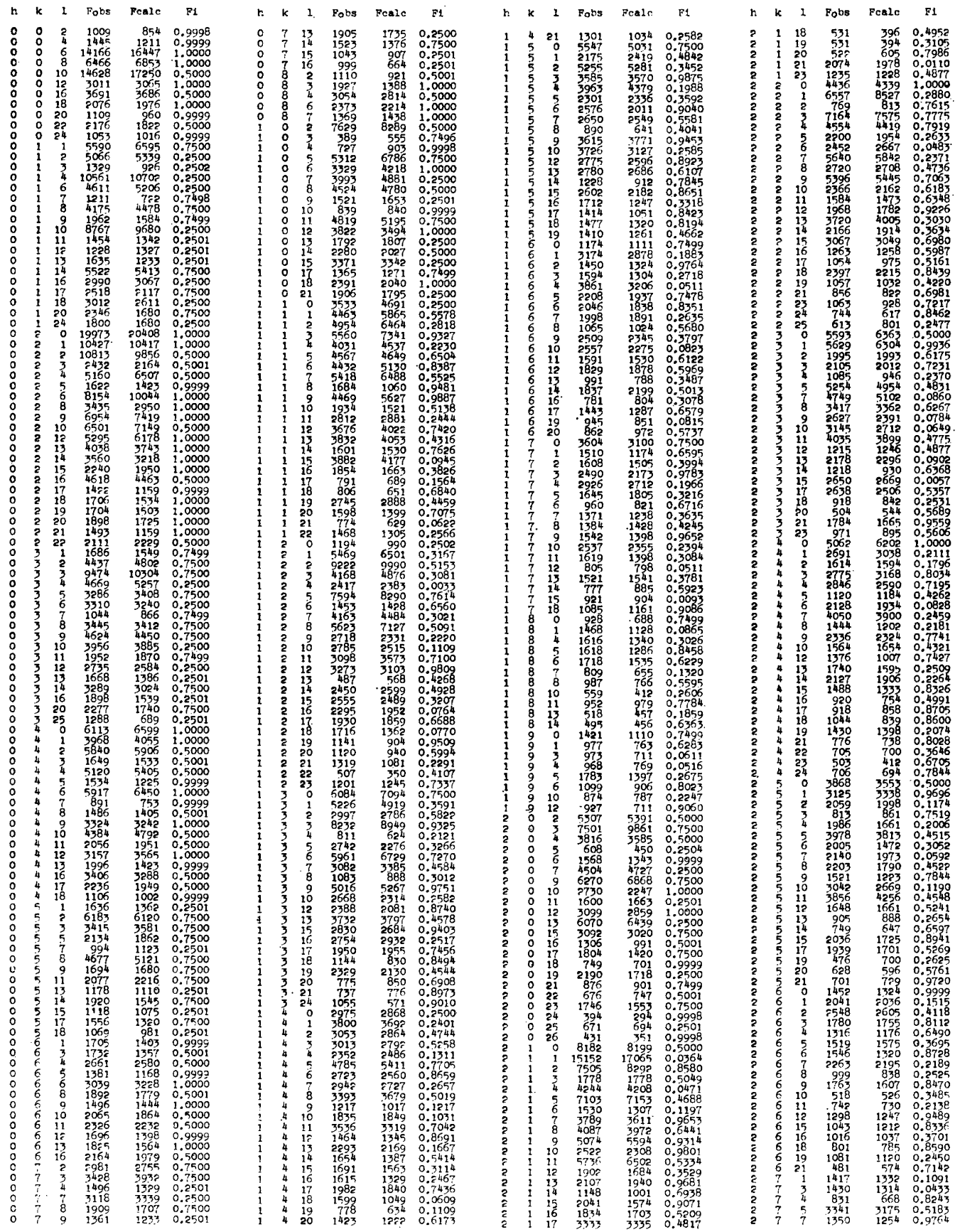

Acta Chem. Scand. 20 (1966) No. 4 
Table 4. Continued.
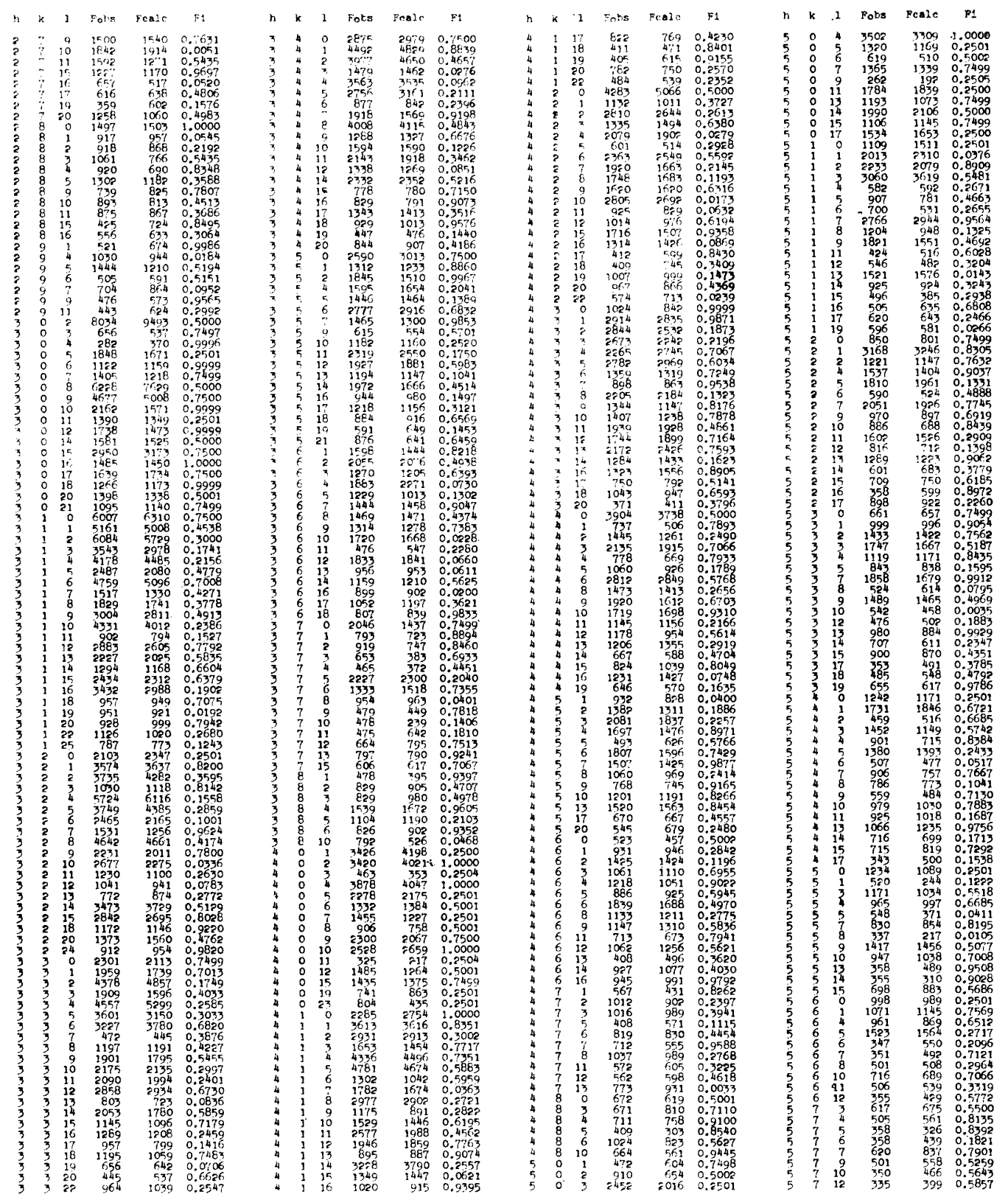

Acta Chem. Scand. 20 (1966) No. 4 
Table 5. Bond lengths and bond angles with standard deviations for the heavier atoms of the molecule.

\begin{tabular}{|c|c|c|}
\hline & $\begin{array}{c}\text { Bond length } \\
(\AA)\end{array}$ & $\sigma(\AA)$ \\
\hline $\mathrm{Brl}-\mathrm{Cl}$ & 1.93 & $(0.02)$ \\
\hline $\mathrm{O} 1-\mathrm{C} 7$ & 1.23 & $(0.02)$ \\
\hline $\mathrm{O} 2-\mathrm{C} 7$ & 1.41 & $(0.02)$ \\
\hline $\mathrm{O} 2-\mathrm{C} 8$ & 1.43 & $(0.02)$ \\
\hline $\mathrm{O} 3-\mathrm{Cl0}$ & 1.47 & $(0.02)$ \\
\hline $\mathrm{O} 3-\mathrm{Cl4}$ & 1.53 & $(0.03)$ \\
\hline $\mathrm{O} 4-\mathrm{Cll}$ & 1.46 & $(0.02)$ \\
\hline $\mathrm{O} 4-\mathrm{C} 16$ & 1.45 & (0.03) \\
\hline $\mathrm{C} 1-\mathrm{C} 2$ & 1.31 & $(0.03)$ \\
\hline $\mathrm{Cl}-\mathrm{C} 6$ & 1.42 & $(0.03)$ \\
\hline $\mathrm{C} 2-\mathrm{C3}$ & 1.43 & $(0.03)$ \\
\hline C3-C4 & 1.31 & $(0.03)$ \\
\hline $\mathrm{C} 4-\mathrm{C} 5$ & 1.42 & $(0.03)$ \\
\hline $\mathrm{C} 4-\mathrm{C} 7$ & 1.54 & $(0.02)$ \\
\hline $\mathrm{C} 5-\mathrm{C} 6$ & 1.41 & $(0.03)$ \\
\hline $\mathrm{C} 8-\mathrm{C} 9$ & 1.65 & $(0.03)$ \\
\hline $\mathrm{C} 8-\mathrm{Cl} 2$ & 1.57 & $(0.03)$ \\
\hline $\mathrm{C} 9-\mathrm{C} 10$ & 1.56 & $(0.03)$ \\
\hline $\mathrm{C} 9-\mathrm{Cl} 3$ & 1.45 & $(0.04)$ \\
\hline $\mathrm{C} 9-\mathrm{C} 15$ & 1.56 & $(0.02)$ \\
\hline $\mathrm{C} 10-\mathrm{C} 11$ & 1.54 & (0.04) \\
\hline $\mathrm{C} 10-\mathrm{C} 14$ & 1.57 & (0.04) \\
\hline $\mathrm{C} 11-\mathrm{Cl} 2$ & 1.54 & $(0.03)$ \\
\hline $\mathrm{C} 15-\mathrm{Cl} 6$ & 1.48 & $(0.03)$ \\
\hline $\mathrm{Cl5}-\mathrm{C} 17$ & 1.50 & $(0.03)$ \\
\hline $\mathrm{C} 15-\mathrm{C} 18$ & 1.65 & (0.03) \\
\hline $\mathrm{C} 16-\mathrm{C} 21$ & 1.49 & $(0.03)$ \\
\hline C18-C19 & 1.69 & $(0.03)$ \\
\hline $\mathrm{C} 19-\mathrm{C} 20$ & 1.43 & $(0.04)$ \\
\hline $\mathrm{C} 20-\mathrm{C} 21$ & 1.27 & $(0.03)$ \\
\hline $\mathrm{C} 20-\mathrm{C} 22$ & 1.54 & $(0.03)$ \\
\hline
\end{tabular}

$\mathrm{C} 7-\mathrm{O} 2-\mathrm{C} 8$

$\mathrm{C10}-\mathrm{O3}-\mathrm{Cl4}$

$\mathrm{C11}-\mathrm{O} 4-\mathrm{Cl} 6$

$\mathrm{Brl}-\mathrm{Cl}-\mathrm{C} 2$

$\mathrm{Br} 1-\mathrm{C} 1-\mathrm{C} 6$

$\mathrm{C} 2-\mathrm{C} 1-\mathrm{C} 6$

$\mathrm{Cl}-\mathrm{C} 2-\mathrm{C} 3$

$\mathrm{C2}-\mathrm{C3}-\mathrm{C} 4$

C3-C4-C5

$\mathrm{C3}-\mathrm{C} 4-\mathrm{C} 7$

$\mathrm{C} 5-\mathrm{C} 4-\mathrm{C} 7$

$\mathrm{C4}-\mathrm{C} 5-\mathrm{C} 6$

$\mathrm{C} 1-\mathrm{C} 6-\mathrm{C} 5$

$\mathrm{O} 1-\mathrm{C} 7-\mathrm{O} 2$

$\mathrm{O} 1-\mathrm{C} 7-\mathrm{C} 4$

$\mathrm{O} 2-\mathrm{C} 7-\mathrm{C} 4$

O2-C8-C9

$\mathrm{O} 2-\mathrm{C} 8-\mathrm{Cl} 2$

$\mathrm{C9}-\mathrm{C} 8-\mathrm{C12}$

$\mathrm{C} 8-\mathrm{C} 9-\mathrm{Cl0}$

C8-C9-C13

C8-C9-Cl5

$\mathrm{C10}-\mathrm{C9}-\mathrm{C} 13$

C10-C9-C15

C13-C9-C15

$\mathrm{O} 3-\mathrm{Cl0}-\mathrm{C} 9$

O3-C10-C11

$\mathrm{O} 3-\mathrm{C10}-\mathrm{C} 14$

C9-C10-C11

$\mathrm{C9}-\mathrm{Cl0}-\mathrm{C14}$

$\mathrm{C} 11-\mathrm{Cl}$-C14

O4-C11-C10

$\mathrm{O} 4-\mathrm{Cl1}-\mathrm{Cl2}$

C10-C11-C12

$\mathrm{C} 8-\mathrm{Cl2}-\mathrm{Cl1}$

$\mathrm{O} 3-\mathrm{C} 14-\mathrm{C} 10$

C9-Cl5-C16

$\mathrm{C} 9-\mathrm{Cl5}-\mathrm{Cl7}$

C9-C15-C18

C16-C15-C17

C16-C15-C18

$\mathrm{C} 17-\mathrm{C} 15-\mathrm{C} 18$

$\mathrm{O} 4-\mathrm{C} 16-\mathrm{C} 15$

O4-C16-C21

$\mathrm{C} 15-\mathrm{C} 16-\mathrm{C} 21$

$\mathrm{C15}-\mathrm{C} 18-\mathrm{Cl9}$

C18-C19-C20

C19-C20-C21

C19-C20-C22

$\mathrm{C} 21-\mathrm{C} 20-\mathrm{C} 22$

C16-C21-C20
Angle $\left({ }^{\circ}\right) \quad \sigma\left({ }^{\circ}\right)$

$\begin{aligned} 116 & (1) \\ 63 & (2) \\ 115 & (2) \\ 121 & (2) \\ 114 & (2) \\ 124 & (2) \\ 119 & (2) \\ 118 & (2) \\ 124 & (2) \\ 122 & (2) \\ 114 & (2) \\ 118 & (2) \\ 116 & \end{aligned}$

116

120

127

112

111

109

104

100

112

101

115

110

117

118

119

60

103

122

129

108

115

99

106

57

114

112

105

112

105

108

113

106

116

104

115

119

111

127

128
(2)

(2)

(2)

(2)

(2)

(2)

(2)

(2)

(2)

(2)

(2)

(2)

(2)

(2)

(2)

(3)

(2)

(2)

(2)

(2)

(2)

(2)

(2)

(2)

(2)

(2)

(2)

(2)

Acta Chem. Scand. 20 (1966) No. 4 
Acknowledgements. We wish to thank Mrs. M. Innes and Mrs. A Johansson for technical assistance. Financial support from the Swedish Natural Science Research Council, the Swedish Medical Research Council, and the U.S. Public Health Service (GM-11653) is gratefully acknowledged.

\section{REFERENCES}

1. Godtfredsen, W. O. and Vangedal, S. Proc. Chem. Soc. 1964188.

2. Godtfredsen, W. O. and Vangedal, S. Acta Chem. Scand. 19 (1965) 1088.

3. Freeman, G. G., Gill, J. E. and Waring, W. S. J. Chem. Soc. 19591105.

4. Fishman, J., Jones, E. R. H., Lowe, G. and Whiting, M. C. J. Chem. Soc. 19603948.

5. Abrahamsson, S. and Nilsson, B. Proc. Chem. Soc. 1964188.

6. Abrahamsson, S., Aleby, S., Larsson, K., Nilsson, B., Selin, K. and Westerdahl, A. Acta Chem. Scand. 19 (1965) 758.

7. Mills, O. S. and Rollett, J. S. Computing Methods and the Phase Problem in X-Ray Crystal Analysis, Pergamon Press, London 1960, p. 107.

8. Gutzwiller, J. and Tamm, Ch. Helv. Chim. Acta 46 (1963) 1786.

9. McPhail, A. T. and Sim, G. A. Chem. Comm. 15 (1965) 350.

10. Ahmed, F. R. and Cruickshank, D. W. J. Acta Cryst 6 (1953) 385.

11. Darlow, S. F. Acta Cryst. 13 (1960) 683.

12. Abrahamsson, S. Acta Cryst. 16 (1963) 409.

Received December 9, 1965. 